\title{
ORÇAMENTO PÚBLICO MUNICIPAL DA CULTURA: FUNÇÃO, SUBFUNÇÃO OU SEM-FUNÇÃO?
}

\author{
MUNICIPAL CULTURAL BUDGET: FUNCTION, SUB FUNCTION OR NO-FUNCTION? \\ PRESUPUESTO PÚBLICO MUNICIPAL DE CULTURA: ¿FUNCIÓN, SUBFUNCIÓN O SIN-FUNCIÓN?
}

\section{RESUMO}

0 debate sobre financiamento público para a cultura ocorre no mundo todo, envolvendo os diversos níveis de governo. No Brasil, enquanto percentuais mínimos de investimento público em cultura permanecem indefinidos, a função cultura nos orçamentos municipais segue dependendo de pressões políticas e negociações pontuais. Considerando o orçamento público um instrumento de planejamento, 0 artigo analisa os dados orçamentários em relação à cultura nos 10 maiores municípios da Região Metropolitana de São Paulo, de 2008 até 2016. Os dados foram extraídos do Portal Meu Município, da Secretaria do Tesouro Nacional e dos portais oficiais dos municípios. Também foram realizadas entrevistas com gestores(as) de organizações culturais do município de São Paulo. Os resultados mostram que há uma dependência grande dos editais de fomento e que a dinâmica orçamentária municipal interfere na atuação dos grupos culturais, que precisam, independentemente do repasse financeiro público, ter sustentabilidade financeira para desempenhar suas atividades.

PALAVRAS-CHAVE: Cultura, orçamento municipal, finanças públicas, organizações culturais, política cultural.

Laura Angélica Moreira Silva ${ }^{1}$

lauraams@gmail.com

ORCID: 0000-0003-1139-0422

Fernando Burgos ${ }^{1}$

fernando.burgos@fgv.br

ORCID: 0000-0002-0910-4127

Anny Karine de Medeiros ${ }^{1}$

annykmedeiros@gmail.com

ORCID: 0000-0002-9208-5720

${ }^{1}$ Fundação Getulio Vargas, Escola de Administração de Empresas de São Paulo, São Paulo, SP, Brasil

Submetido 15.06.2018. Aprovado 23.11.2018

Avaliado pelo processo de double blind review. Editores convidados: Arnaldo Mauerberg Jr., José Roberto Afonso e Sol Garson.

DOI: http://dx.doi.org/10.12660/cgpc.v23n76.75426 


\section{ABSTRACT}

The debate over public funding for culture occurs worldwide, involving various governmental levels. In Brazil, while minimum percentages of public investment in culture remain undefined, the culture function in municipal budgets remains dependent on political pressures and particular negotiations. Considering the public budget as a planning tool, the article analyzes the budgetary data related to culture in the ten largest municipalities of the Metropolitan Region of São Paulo, from 2008 to 2016 . Methodologically, the data comes from the Portal Meu Município, Secretariat of the National Treasury and official municipal websites. Interviews were also conducted with managers of cultural organizations in the city of São Paulo. The results show that there is great dependence on government funding programs and that the municipal budgetary dynamics interferes with the performance of cultural groups that need, regardless of public financial transfers, financial sustainability to carry out their activities.

KEYWORDS: Culture, municipal budget, public finance, cultural organizations, cultural policy.

\section{RESUMEN}

El debate sobre el financiamiento público para la cultura es mundial, involucrando varios niveles gubernamentales. En Brasil, aunque los porcentajes mínimos de inversión pública en cultura siguen sin definirse, la función de cultura en los presupuestos municipales sigue dependiendo de presiones políticas y negociaciones particulares. Teniendo en cuenta el presupuesto público como una herramienta de planificación, el artículo analiza los datos presupuestarios relacionados con la cultura en los diez municipios más grandes de la Región Metropolitana de São Paulo, de 2008 a 2016. Metodológicamente, los datos provienen del Portal Meu Município, Secretaría de la Tesoro Nacional y sitios web municipales oficiales. Además, hemos realizado entrevistas con gerentes de organizaciones culturales en la ciudad de São Paulo. Los resultados muestran que existe una gran dependencia de los programas de financiamiento del gobierno y que la dinámica presupuestaria municipal interfiere en el desempeño de los grupos culturales que necesitan, independientemente de las transferencias financieras públicas, tener sostenibilidad financiera para llevar a cabo sus actividades.

PALABRAS CLAVE: Cultura, presupuesto municipal, finanzas públicas, organizaciones culturales, política cultural.

\section{INTRODUÇÃO}

Do ponto de vista econômico, cultura é compreendida como uma externalidade positiva, pois pode gerar resultados econômicos não esperados e que são interessantes para o desenvolvimento de determinado território (Einarsson, 2008). O reconhecimento de que as cidades possuem iniciativas a favor do desenvolvimento é, neste trabalho, entendido como um consenso, incluindo-se nessas iniciativas as atividades culturais, que ganharam importância nos últimos anos, dada sua contribuição na resolução de desafios presentes no cotidiano urbano.

Para além disso, as políticas culturais são entendidas como uma forma de viabilizar e garantir o direito à cultura, tal qual preconizado pela Declaração Universal dos Direitos
Humanos (Organização das Nações Unidas, 1948). Nesse sentido, essas políticas têm papel fundamental na garantia da cultura como direito. No caso das metrópoles, manifestações culturais que possuem caráter simbólico, transversal e polissêmico podem utilizar os grandes centros urbanos como ferramenta de apresentação e venda de seus trabaIhos. Em muitos locais, essas manifestações podem, ainda, ser instrumento para alcançar determinados objetivos sociais, como apropriação e ressignificação do espaço público, diminuição de violência, atendimento de crianças e adolescentes excluídos socialmente ou melhoria da qualidade de vida de moradores, entre outros.

Apesar desses benefícios citados, há estudos que afirmam existir pouco apoio institucional para as iniciativas culturais, sendo sua sus- 


\section{ORÇAMENTO PÚBLICO MUNICIPAL DA CULTURA: FUNÇÃO, SUBFUNÇÃO OU SEM-FUNÇÃO?}

tentação realizada, principalmente, a partir da iniciativa dos próprios atores e disposição das redes de relações informais entre seus moradores, o que alimenta o debate sobre o financiamento público para a cultura e para políticas culturais (Bille \& Shulze, 2006; Einarsson, 2008; Frey, 1995; Gibson \& Stevenson, 2004; Håkonsen \& Løyland, 2016; Rizzo \& Throsby, 2006). No Brasil, esse debate é trazido por Barbosa da Silva (2004) e Machado, Rabelo e Moreira (2014) ou aparece de maneira tangencial em outros trabalhos (Michetti, 2016; Rubim, 2013).

O presente artigo não tem aspirações de discutir o que é cultura e quais os seus objetivos na sociedade, mas utiliza esse setor como recorte analítico por entender que o campo da cultura envolve constante disputa. Essa disputa se reflete em distintos modelos de políticas culturais, bem como na destinação de recursos para tais políticas. Isso porque, importa enfatizar, a distribuição orçamentária não pode ser reduzida a uma decisão meramente técnica. A distribuição do orçamento perpassa questões políticas e de economia política, o que envolve um jogo de poder e um conflito distributivo. Dessa forma, reforça-se o objetivo de trazer para o debate o orçamento público, como este é gerido e quais os mecanismos de financiamento passíveis de uso. $\mathrm{O}$ recorte sobre recursos da cultura é uma opção metodológica do artigo, ao considerar que esses recursos são historicamente espaço de disputa de poder entre diferentes posições, não tendo uma destinação mínima estabelecida por lei - ao contrário de áreas como educação e saúde -, mas frequentemente alvo de pressões nacionais e internacionais, via Unesco, por exemplo, para aumento dos recursos destinados à área.
Como forma de ampliar o debate sobre o financiamento cultural, o presente artigo visa analisar a importância dos mecanismos de repasse de recursos municipais para cultura, dado que a busca pela sustentabilidade financeira dos grupos culturas é um enorme desafio. Nos últimos anos, editais de fomento à cultura colaboraram para que organizações culturais conseguissem desempenhar suas atividades. Tal repasse pode ocorrer por programas federais, estaduais e municipais.

No entanto, com a atual crise econômica brasileira, tem-se por hipótese deste trabalho que o recurso disponível por meio dessa ferramenta de fomento tem sido reduzido. Com menor oferta de recursos, o processo, para ser contemplado por algum edital, torna-se cada vez mais concorrido. Quando vencida essa etapa, outro problema pode surgir para as organizações culturais: o atraso no repasse de recursos. Esse atraso pode estar vinculado às inúmeras dificuldades financeiras por que as administrações públicas estão passando, e uma das estratégias utilizadas pelo Poder Executivo é utilizar o mecanismo de limitar o empenho (ato de criar a obrigação de realizar um pagamento) das despesas, bem como as movimentações financeiras de uma prefeitura. Essa ação é também conhecida como "contingenciamento".

O problema é que, em muitas áreas, incluindo a cultura, o contingenciamento de recursos transforma-se em contingenciamento das atividades programadas para aquele período. Assim, mesmo quando contempladas pelos editais, as organizações enfrentam dificuldades que provocam adiamento das atividades, interrupções de algumas delas 
ou transformações nas organizações, que passam a buscar alternativas para manter o seu funcionamento regular.

O artigo está dividido nesta introdução, em uma breve explicação sobre a abordagem metodológica, para, então, tratar das interfaces da cultura com a economia e do orçamento como instrumento de planejamento e execução de atividades culturais, permitindo analisar a despesa com a função cultura nos municípios objetos da presente pesquisa. A análise dos valores previstos e liquidados colabora para compreender a dinâmica orçamentária e seus efeitos nas organizações culturais, levando às considerações finais do presente artigo.

\section{ABORDAGENS METODOLÓGICAS}

Para a realização deste artigo, utilizamos duas abordagens. A primeira delas foi a análise de dados secundários relativos ao orçamento municipal de cultura no estado de São Paulo. A partir das dificuldades encontradas para localizar os valores referentes à função cultura, optou-se por aprofundar a análise nos 10 municípios mais populosos da Região Metropolitana de São Paulo (RMSP), conforme a Tabela 1. Embora haja diferenças importantes de extensão territorial, população, densidade demográfica, Produto Interno Bruto (PIB) e IDH em toda a região metropolitana, o critério de escolha considerou que o contingente populacional poderia gerar maior número de organizações culturais, e também um maior público-alvo a ser atendido pelos programas definidos pelas prefeituras.

Tabela 1. Municípios com maior população

\begin{tabular}{|c|c|c|c|c|c|}
\hline Municípios & $\begin{array}{l}\text { Área } \\
\left(\mathrm{km}^{2}\right)\end{array}$ & $\begin{array}{c}\text { População em } \\
2017\end{array}$ & $\begin{array}{c}\text { Densidade } \\
\text { demográfica } \\
\left(\mathrm{hab} / \mathrm{km}^{2}\right)\end{array}$ & $\begin{array}{l}\text { PIB } 2014 \text { (mil } \\
\text { reais) }\end{array}$ & $\begin{array}{c}\text { IDH- } \\
-\mathrm{M} \text { em } \\
2010\end{array}$ \\
\hline São Paulo & $1.521,11$ & 12.106.920 & $8.006,6$ & $\mathrm{R} \$ 628.064 .882$ & 0,805 \\
\hline Guarulhos & 318,68 & 1.349 .113 & $4.233,4$ & $\mathrm{R} \$ 51.389 .524$ & 0,763 \\
\hline São Bernardo do Campo & 409,53 & 827.437 & $2.020,5$ & $\mathrm{R} \$ 47.551 .620$ & 0,805 \\
\hline Santo André & 175,78 & 715.231 & $4.068,9$ & $\mathrm{R} \$ 28.119 .591$ & 0,815 \\
\hline Osasco & 64,95 & 697.886 & $10.745,0$ & $\mathrm{R} \$ 58.566 .199$ & 0,776 \\
\hline Mauá & 61,91 & 462.005 & $7.462,5$ & $\mathrm{R} \$ 11.329 .503$ & 0,766 \\
\hline Mogi das Cruzes & 712,54 & 433.901 & 608.9 & $\mathrm{R} \$ 13.367 .335$ & 0,783 \\
\hline Diadema & 30,73 & 417.869 & $13.598,1$ & $\mathrm{R} \$ 13.910 .517$ & 0,757 \\
\hline Carapicuíba & 34,54 & 396.587 & $11.482,0$ & $\mathrm{R} \$ 4.719 .835$ & 0,749 \\
\hline Itaquaquecetuba & 82,62 & 360.657 & $4.365,3$ & $\mathrm{R} \$ 5.742 .348$ & 0,714 \\
\hline
\end{tabular}

Fonte: Empresa Paulista de Planejamento Metropolitano (Emplasa), GIP/CDI, 2017. 
A Figura 1 mostra o mapa da RMSP, com destaque para os municípios que foram analisados. É importante ressaltar que a interconexão, via rede de transporte público metropolitano, faz com que as pessoas circulem entre as cidades da região para trabalhar, fazer compras, relacionar-se com parentes e amigos, frequentar espaços de convívio e de lazer e também para acessar ações culturais. Ao mesmo tempo, as organizações culturais circulam entre as diferentes cidades de acordo com a disponibilidade de recursos para a realização dessas mesmas ações culturais. Nesse cenário, em que pese a desproporção da cidade de São Paulo com relação às demais, seu papel como ca- talisador de ações e fomentador, via editais, torna essencial sua consideração na análise de políticas culturais em grandes centros urbanos. É assim na capital paulista, mas o mesmo fenômeno de atração da população da cidade vizinha também ocorre em outras regiões metropolitanas brasileiras, como Rio de Janeiro, Recife, Salvador ou Belém, por exemplo. O papel do município de São Paulo como fomentador da cultura foi um elemento que apareceu nas entrevistas realizadas com coletivos culturais, que participaram de editais de seleção para ações culturais na região, tendo o programa VAI da prefeitura de São Paulo como ponto comum.

Figura 1. Mapa da Região Metropolitana de São Paulo com os municípios analisados em destaque

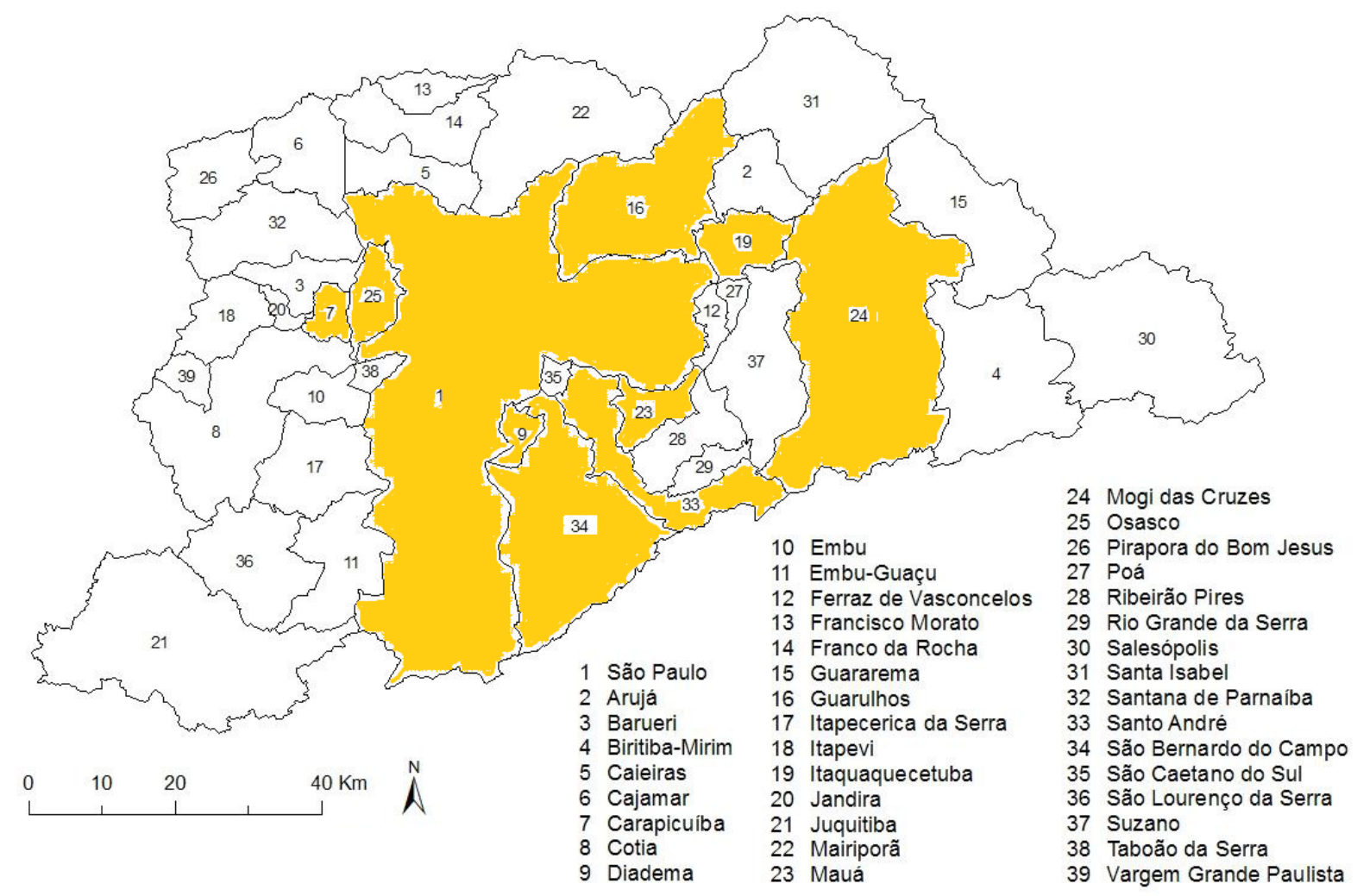


Posteriormente, buscou-se, nos portais oficiais das Prefeituras, bem como nos portais oficiais das Câmaras de Vereadores, informações orçamentárias para que fosse realizado um primeiro filtro com foco na área da cultura. Buscaram-se dados sobre o que se esperava gastar na função cultura de cada município e o que efetivamente foi gasto por meio da variável liquidação, no período de 2008 a 2016. Esses dados são referentes ao planejamento realizado pelas Prefeituras, bem como a liquidação realizada por estas, que é uma das etapas que compõem a despesa orçamentária, tal como previsto na legislação que determina as normas gerais de direito financeiro (Lei n. 4.320, 1964). Tal análise possui o intuito de observar o comportamento orçamentário no setor cultural e a capacidade das Prefeituras em questão de manter o orçamento planejado e liquidá-lo da forma como se espera. Para essa análise, foram utilizados os dados presentes na chamada função cultura. É de conhecimento a existência de subfunções dentro da função cultura, contudo nem todos os municípios analisados descrevem suas despesas de maneira tão detalhada. Assim, as subfunções foram expurgadas na análise.

Diante da dificuldade de encontrar, nos portais oficiais das Prefeituras, os dados de prestação de contas das suas atividades, a descrição e construção de inferências foi feita por meio de dados secundários extraídos da base Finanças do Brasil (Finbra) presente no portal oficial da Secretaria do Tesouro Nacional. Essa base refere-se à prestação de contas que todo ente federado deve executar no fim do exercício. Já as peças orçamentárias vinculadas ao planejamento orçamentário, a saber, a Lei de Diretrizes Orçamentárias (LDO) e a Lei Orçamentária Anual (LOA), foram extraídas dos portais oficiais dos municípios. Contudo, também nessa etapa houve dificuldades para garantir que todas as peças fossem analisadas perante a liquidação apresentada para a Secretaria do Tesouro Nacional. De maneira sistematizada, as principais dificuldades encontradas estão no Quadro 1: 


\section{ORÇAMENTO PÚBLICO MUNICIPAL DA CULTURA: FUNÇÃO, SUBFUNÇÃO OU SEM-FUNÇÃO?}

Quadro 1. Principais dificuldades encontradas na coleta dos dados

\begin{tabular}{|l|l|}
\hline \multicolumn{1}{|c|}{ Municípios } & \multicolumn{1}{c|}{ Dificuldades } \\
\hline São Paulo & $\begin{array}{l}\text { As informações de despesas estão previstas na Lei Orçamentária } \\
\text { Anual (LOA) desagregadas por subfunção. a partir do ano de 2014. } \\
\text { Além disso a base da LOA conta com algumas subfunções não pre- } \\
\text { sentes no Finbra. que agrupa em sua metodologia esses dados em } \\
\text { uma categoria intitulada de "outras subfunções." Essas ocorrências } \\
\text { (subfunções: 126. 131. 422. 452. 695) não foram incluídas neste traba- } \\
\text { Iho dado que não ocorrem em todos os anos. apenas no final. }\end{array}$ \\
\hline Guarulhos & $\begin{array}{l}\text { Indisponibilidade despesa por categoria funcional programática na } \\
\text { LOA de 2012. }\end{array}$ \\
\hline São Bernardo do Campo & Indisponibilidade da LOA de 2008. \\
\hline Santo André & Disponibilidade de informações para os anos de 2014 e 2015. \\
\hline Osasco & $\begin{array}{l}\text { Para os anos de 2013 a 2016 foi utilizado o documento "Demonstrativo } \\
\text { por Funções. Programas e Subprogramas por Projetos e Atividades". }\end{array}$ \\
\hline Mauá & Indisponibilidade das LOAs de 2008 e 2009. \\
\hline Mogi das Cruzes & $\begin{array}{l}\text { Indisponibilidade de todas as LOAs do período. assim como de qual- } \\
\text { quer outro documento que registre as despesas do município. }\end{array}$ \\
\hline Carapicuíba & $\begin{array}{l}\text { Indisponibilidade das LOAs anteriores a 2011. Na LOA de 2012 não foi } \\
\text { encontrado o anexo das despesas por função. como nos outros anos. }\end{array}$ \\
\hline Itaquaquecetuba & Indisponibilidade das LOAs de 2008 e 2009. \\
\hline
\end{tabular}

Conforme o quadro, chama atenção a quantidade de municípios da RMSP cujas informações não são facilmente disponibilizadas. Apesar de não ser objetivo do presente artigo, impressiona a falta de transparência com os dados de finanças públicas. Para o município de Carapicuíba, por exemplo, a LOA não foi encontrada para os anos de 2008, 2009, 2010 e 2012, o que dificulta a análise clara e correta sobre o planejamento do município e sua efetiva ação na política pública de cultura. Além disso, sua receita não está registrada no Portal Meu Município, fonte de dados para essa variável.

Com base em Einarsson (2008), que afirma que as despesas de cultura são determinadas nos orçamentos dos níveis central e local e que elas possuem impacto significativo nas atividades culturais, a segunda abordagem metodológica foi qualitativa. $\mathrm{Na}$ busca por entender o impacto que os recursos públicos geram nas organizações culturais, foram realizadas 12 entrevistas com coletivos culturais que atuam prioritariamente na zona sul do município de São Paulo, entre março de 2017 e janeiro de 2018. Os coletivos entrevistados foram apoiados pelo Programa para a Valorização de Iniciativas Culturais (VAI), projeto da Prefeitura de São Paulo implantado em 2003 com a finalidade de apoiar financeiramente projetos culturais, o programa apoia, em sua maioria, projetos de jovens de baixa renda e de regiões mais carentes da cidade, pois estes possuem maiores dificuldades em manter a susten- 
tabilidade das atividades desenvolvidas. O programa funciona por meio de editais, abertos anualmente pela Secretaria Municipal de Cultura.

\section{AS INTERFACES DA CULTURA COM A ECONOMIA}

Para Einarsson (2008), cultura é comportamento ou atividade humana que é passada adiante e que se apresenta em forma de emoções e comportamentos sociais. Além disso, o autor apresenta a noção de bem público e a controvérsia existente entre cultura como bem público e, ao mesmo tempo, cultura como bem excludente, quando se analisam bens culturais que estão direcionados a determinados nichos da sociedade que possuem acesso (em muitos casos, por meio de recursos financeiros) a atividades culturais consideradas elitizadas, tais como concertos e exposições vinculadas às artes plásticas. Na tentativa de ampliar o acesso a diferentes formatos de produtos culturais, a administração pública tende a interferir no setor, desenhando políticas de incentivo à promoção da cultura. Algumas ações públicas podem, inclusive, interferir positivamente em outras áreas, como educação e desenvolvimento local.

No que tange à interface com o desenvolvimento local, cabe registrar o que se compreende não somente por cultura, mas também por economia da cultura. Esse termo reflete uma ou mais atividades de cunho cultural capazes de gerar lucros para o indivíduo e, de certa forma, se tornar um ativo (re)conhecido. Nesse sentido, a economia da cultura pode vir a ultrapassar as discussões de fomento vinculadas ao apoio no restauro de patrimônio histórico arquitetônico, preserva- ção de acervos ou apoio à produção cinematográfica, formas mais conservadoras de política cultural.

O exercício de se pensar e definir o que vem a ser a economia da cultura não faz parte de um esforço recente. Uma correlação comumente feita é aquela que incorpora à ideia de economia da cultura o termo economia criativa. A publicação The Economy of Culture in Europe, utilizada por Mello, Gorgulho, Goldenstein e Alexandre (2009), coloca a necessidade de utilizar dois conceitos para, ao mesmo tempo, vincular e desvincular as ideias que ambos estabelecem. $\mathrm{O}$ primeiro desses conceitos é o de "setor cultural", entendido como espaço capaz de gerar parâmetros para a produção artística, seja para consumo imediato ou para reprodução, como livros, pinturas, entre outros, além da produção cultural em massa, como a música e o cinema. O segundo conceito é o de "setor criativo", estando a diferença na cultura como "um insumo para a produção de bens funcionais, não culturais" (Mello et al., 2009, p. 302), ultrapassando a ideia de arte, absorvendo valores e hábitos dos indivíduos que são traduzidos, por exemplo, na publicidade, na moda e no design.

Uma definição adicional de bens e serviços culturais é proposta por Throsby (2010), sendo derivada de uma consideração do tipo de valor que eles incorporam ou geram. Assim, os bens e serviços culturais possuem valor cultural além de qualquer valor comercial que possam possuir, e esse valor cultural pode não ser totalmente mensurável em termos monetários. Assim, os bens e serviços culturais são valorizados tanto por aqueles que os fabricam como por aqueles que os consomem, por razões sociais e culturais 


\section{ORÇAMENTO PÚBLICO MUNICIPAL DA CULTURA: FUNÇ̃̃o, SUBFUNÇ̃̃o OU SEM-FUNÇÃo?}

que podem complementar ou transcender uma avaliação puramente econômica. Essas razões podem incluir preocupações espirituais, considerações estéticas ou a contribuição dos bens e serviços para a compreensão da identidade cultural da comunidade. Se tal valor cultural puder ser identificado, pode servir como uma característica observável para distinguir os tipos de bens culturais de outros tipos de commodities.

Mas Throsby (2010) aponta que, qualquer que seja a definição, os bens e serviços culturais podem ser vistos como um subconjunto de uma categoria mais ampla de bens que podem ser chamados de bens e serviços criativos. Esses últimos são simplesmente produtos que requerem algum nível razoavelmente significativo de criatividade em sua fabricação, sem necessariamente satisfazer outros critérios que lhes permitam ser rotulados como "culturais". Assim, a categoria "bens criativos" se estende para além dos bens culturais, incluindo produtos como propaganda e software; esses últimos bens e serviços podem ser vistos como produtos essencialmente comerciais, mas, conforme o autor, envolvem algum nível de criatividade em sua produção.

Ambos os setores podem ser, então, entendidos como cadeias produtivas com probabilidade de gerar crescimento econômico ou retorno financeiro e são interdependentes, dado que se beneficiam das externalidades do outro setor. Essas externalidades positivas são frequentemente mencionadas em estudos sobre os ganhos econômicos oriundos do setor cultural. Kuti e Marschall (1992) apontam que os bens culturais podem beneficiar muitas pessoas que não os conso- mem diretamente. Os autores explicam que uma cidade, ao decidir investir em um museu, poderá gerar benefícios a uma grande parcela da população, dado que os turistas atraídos pelo museu podem fazer refeições ou se hospedar nos hotéis da cidade. Isso gerará empregos e um aumento de arrecadação de impostos e taxas municipais. Esse incremento de receita, por sua vez, poderá ser investido em equipamentos de lazer que podem beneficiar a população local que nunca visitou o museu nem tem interesse em visitá-lo.

Em outro exemplo importante, Throsby (2010) mostra que um prédio histórico ou uma região caracterizada como patrimônio cultural pode dar origem a uma externalidade benéfica se os transeuntes tiverem prazer em observar suas qualidades estéticas ou históricas. $\mathrm{O}$ autor afirma que pessoas andando por uma cidade como Roma ou Paris podem apreciar a vista dos edifícios históricos, monumentos e praças que encontram. Em princípio, o valor econômico de tal benefício poderia ser estimado, apesar de raramente ser, mas, mesmo assim, os efeitos positivos podem ser identificados e agregarem um valor significativo que a preservação do patrimônio traz aos indivíduos.

O autor também traz a ideia das "externalidades de rede" ou "externalidades de aglomeração", que ocorrem nas indústrias culturais quando as empresas se localizam próximas umas das outras e desfrutam de benefícios recíprocos de outras empresas envolvidas na produção de produtos culturais semelhantes. Essas externalidades ajudariam a explicar o crescimento de centros de produção cultural como Hollywood ou a indústria da moda em Milão (Throsby, 2010). 
O exemplo da indústria cinematográfica californiana e o papel dos bens culturais locais também aparece em Scott (1997).

A compreensão da dimensão econômica da cultura é importante para que, ao analisar como o poder público define o orçamento para a cultura, seja possível aproximarmos de uma tomada de decisão: Busca-se induzir o mercado cultural local já existente ou busca-se interferir em realidades e contextos de vulnerabilidade preexistentes? Assim, infere-se previamente que o poder público compreende a cultura ora como o trabalho que gera um significado social, ora como o trabalho que possui significado econômico.

Rizzo e Throsby (2006) mostram que o processo de decisão de alocação de recursos varia dependendo da área do setor cultural e apontam que, no caso do patrimônio cultural, há diversos outros elementos envolvidos nessa decisão. Ao analisarem os serviços culturais fornecidos por municípios noruegueses, Håkonsen e Løyland (2016) dividiram em oito tipos: atividades para crianças e jovens, bibliotecas, cinemas, museus, divulgação artística, patrimônio cultural, escolas culturais e outros serviços culturais. Os resultados deixam claro que "os gastos culturais locais" não são um fenômeno homogêneo, portanto, em geral, não são afetados de maneira uniforme pelas variáveis econômicas, demográficas ou políticas.

Ainda sob o viés institucional de compreensão da cultura e suas interfaces, o Ministério da Cultura (2016) reconhece a cultura como elemento estratégico para o desenvolvimento econômico, dado que muitas atividades culturais ganham essa perspectiva. Desse modo, reforça-se aqui a necessidade de ob- servar se o recurso na função cultura prevista nos orçamentos públicos está sendo resguardado para atingir os fins presentes nas competências de cada Secretaria Municipal da RMSP.

\section{O ORÇAMENTO COMO INSTRUMENTO DE PLANEJAMENTO PARA A CULTURA}

O texto clássico de Musgrave (1959) estabelece três funções para o orçamento: alocativa, distributiva e estabilizadora. Em relação à função alocativa, a que se relaciona diretamente com o artigo, Giambiagi e Além (2010) esclarecem que, mesmo que o Estado não seja responsável pela produção de um bem ou serviço, "ele é intrinsicamente responsável pela sua provisão, isto é, deve zelar - diretamente ou através dos órgão reguladores - para que a população seja adequadamente servida em termos da oferta e da qualidade de certos bens ou serviços" (p. 13).

Para Giacomoni (2010), o orçamento deve ser entendido como um sistema: "Ao contrário do que acontecia no passado, hoje o orçamento deve ser visto como parte de um sistema maior, integrado por planos e programas de onde saem as definições e os elementos que vão possibilitar a própria elaboração orçamentária" (p. 206). Assim, ao elaborar planos e programas - ou planejá-los - os tomadores de decisão estão também elaborando a peça orçamentária.

Frey (1994) lembra que, em uma democracia, ao eleger o parlamento e os governantes, os eleitores também estão decidindo sobre a alocação de recursos para a arte. $\mathrm{O}$ autor lembra que existem muitas áreas e instâncias nas quais os indivíduos não exercem sua demanda por arte diretamente, mas 


\section{ORÇAMENTO PÚBLICO MUNICIPAL DA CULTURA: FUNÇÃO, SUBFUNÇÃO OU SEM-FUNÇÃO?}

deixam as decisões para algum órgão representativo. Este pode ser o parlamento (que determina o tamanho do orçamento destinado às artes), a administração pública (responsável pela distribuição dos fundos para as várias instituições de arte e artistas) ou um grupo de especialistas (que podem exercer controle sobre as relações existentes ou distribuir o dinheiro na forma de prêmios ou salários).

Ao estabelecer uma análise histórica sobre os orçamentos dos governos, Premchand (1983) aponta que, inicialmente, o processo orçamentário estava ligado à accountability dos legisladores, em uma disputa com os monarcas. A partir do momento em que conseguiram o direito de impor, alterar ou aprovar as propostas fiscais, os legisladores voltaram sua atenção para o controle de gastos. E afirma que "The legislative accountability inherent in these principles continues to govern the budget today. In addition, the budget has acquired dimensions of planning and management and has become a key instrument of national policymaking" (Premchand, 1983, p. 36).

Em muitas áreas sociais, como saúde e educação, a implementação das políticas e dos programas é, em sua maioria, realizada diretamente pelos governos municipal, estadual e federal. No caso da cultura, essa implementação, em grande medida, é realizada por organizações externas ao Estado, como grupos culturais, ONGs e coletivos. Assim, ao definir planos e programas, os órgãos públicos de cultura (ministérios, secretarias exclusivas e secretarias não exclusivas) estão definindo não apenas a sua forma de atuação, mas também como as organizações culturais devem agir. Ao planejarem suas ações culturais e seus investimentos, os governos estão interferindo direta ou indiretamente no planejamento das ações e atividades das organizações que atuam com cultura.

No Brasil, nos últimos anos, diversos editais de fomento e prêmios foram criados pelos órgãos governamentais de cultura. Em certa medida, para garantir sua sustentabilidade financeira, artistas e organizações criaram novas atividades e/ou alteraram suas formas de trabalhar, de maneira a estarem enquadrados nas regras definidas pelos governos. Isso mostra como o orçamento público de cultura tem um caráter interno - de planejamento público das políticas, programas, projetos e atividades - e também um caráter externo, pois pautam as atuações das organizações.

\section{A DESPESA COM A FUNÇÃO CULTURA NOS MUNICÍPIOS SELECIONADOS}

A base de dados foi construída de 2008 até 2016, mas, conforme mencionado, há muitos dados inexistentes. Por isso, a Tabela 2 diz respeito à evolução do orçamento municipal da área de cultura de 2014 a 2016, nos municípios em que as informações estavam disponíveis: 
Laura Angélica Moreira Silva - Fernando Burgos - Anny Karine de Medeiros

Tabela 2. Dotação orçamentária da Função Cultura entre 2014 e 2016 (em R $\$$ de 2016)

\begin{tabular}{lcccc}
\hline \multicolumn{1}{c}{ Município } & $\mathbf{2 0 1 4}$ & $\mathbf{2 0 1 5}$ & $\mathbf{2 0 1 6}$ & $\begin{array}{c}\text { Variação } \\
\text { no período }\end{array}$ \\
\hline São Paulo & $421.024 .606,25$ & $472.561 .171,29$ & $526.077 .125,70$ & $25,0 \%$ \\
Guarulhos & $38.476 .250,00$ & $24.384 .062,10$ & $17.853 .150,00$ & $-53,6 \%$ \\
São Bernardo do Cam- & & & & \\
po & $59.278 .750,00$ & $52.282 .620,00$ & $43.284 .150,00$ & $-27,0 \%$ \\
Santo André & $40.161 .250,00$ & $26.892 .450,00$ & $26.066 .250,00$ & $-35,1 \%$ \\
Osasco & $10.292 .764,00$ & $12.059 .477,00$ & $12.293 .291,00$ & $19,4 \%$ \\
Mauá & $4.650 .000,00$ & $2.793 .960,00$ & $4.030 .950,00$ & $-13,3 \%$ \\
Diadema & $19.750 .110,00$ & $14.639 .472,90$ & $13.296 .150,00$ & $-32,7 \%$ \\
Carapicuíba & $3.371 .875,00$ & $3.331 .341,00$ & $3.413 .550,00$ & $1,2 \%$ \\
Itaquaquecetuba & $6.337 .883,75$ & $5.375 .448,00$ & $4.334 .400,00$ & $-31,6 \%$ \\
\hline Fon
\end{tabular}

Fonte: Finbra. Os valores foram deflacionados a partir do Índice Nacional de Preços ao Consumidor Amplo (IPCA) do Instituto Brasileiro de Geografia e Estatística (IBGE).

Os dados da Tabela 2 mostram que há três grupos distintos. Entre os municípios cuja previsão orçamentária aumentou no período, estão apenas São Paulo (25,0\%) e Osasco $(19,4 \%)$. O segundo grupo é composto apenas por Carapicuíba, cujo orçamento permaneceu praticamente inalterado, com uma pequena oscilação positiva de $1,2 \%$. O terceiro grupo é composto pelos municípios cujo orçamento da função cultura sofreu redução no período de 2014 a 2016. O destaque mais negativo é para Guarulhos, cujo orçamento da função foi reduzido para menos do que a metade, em valores reais.

A Tabela 3 apresenta a evolução da execução orçamentária total dos municípios entre 2014 e 2016, onde podemos observar que os valores crescem, apesar da grande crise econômica brasileira. Isso mostra que a redução da dotação orçamentária da cultura apresentada anteriormente não seguiu o mesmo padrão da totalidade dos gastos municipais.

Tabela 3. Execução orçamentária liquidada total dos municípios entre 2014 e 2016 (em miIhares de reais de 2016)

\begin{tabular}{lcccc}
\hline \multicolumn{1}{c}{ Município } & $\mathbf{2 0 1 4}$ & $\mathbf{2 0 1 5}$ & $\mathbf{2 0 1 6}$ & $\begin{array}{c}\text { Variação } \\
\text { no período }\end{array}$ \\
\hline São Paulo & 41.660 .125 & 44.227 .102 & 47.997 .559 & $15 \%$ \\
Guarulhos & 3.273 .564 & 3.514 .923 & 3.464 .997 & $6 \%$ \\
São Bernardo do Campo & 3.382 .501 & 3.432 .379 & 3.576 .213 & $6 \%$ \\
Santo André & 1.935 .344 & 2.117 .971 & 2.276 .678 & $18 \%$ \\
Osasco & 1.784 .296 & 1.831 .465 & 1.987 .790 & $11 \%$ \\
Mauá & 789.549 & 831.528 & 923.586 & $17 \%$ \\
Diadema & 976.493 & 1.043 .345 & 1.194 .881 & $22 \%$ \\
Carapicuíba & 418.128 & n.d. & n.d. & n.d. \\
Itaquaquecetuba & 468.744 & 517.142 & 610.404 & $30 \%$ \\
\hline
\end{tabular}

Fonte: Portal Meu Município. Os valores foram deflacionados a partir do IPCA do IBGE. 
A comparação entre as despesas totais na função cultura e as despesas totais do município ao longo dos anos (Gráfico 1) mostra que a maioria dos municípios teve queda no percentual correspondente à cultura entre
2014 e 2016. As exceções ficam para São Paulo, que teve $1,01 \%$ em 2014, 1,07\% em 2015 e 1,10\% em 2016, e Osasco, que passou de $0,58 \%$ para $0,62 \%$.

Gráfico 1. Percentual das despesas na Função Cultura sobre as despesas totais por ano

Fonte: Portal Meu Município.

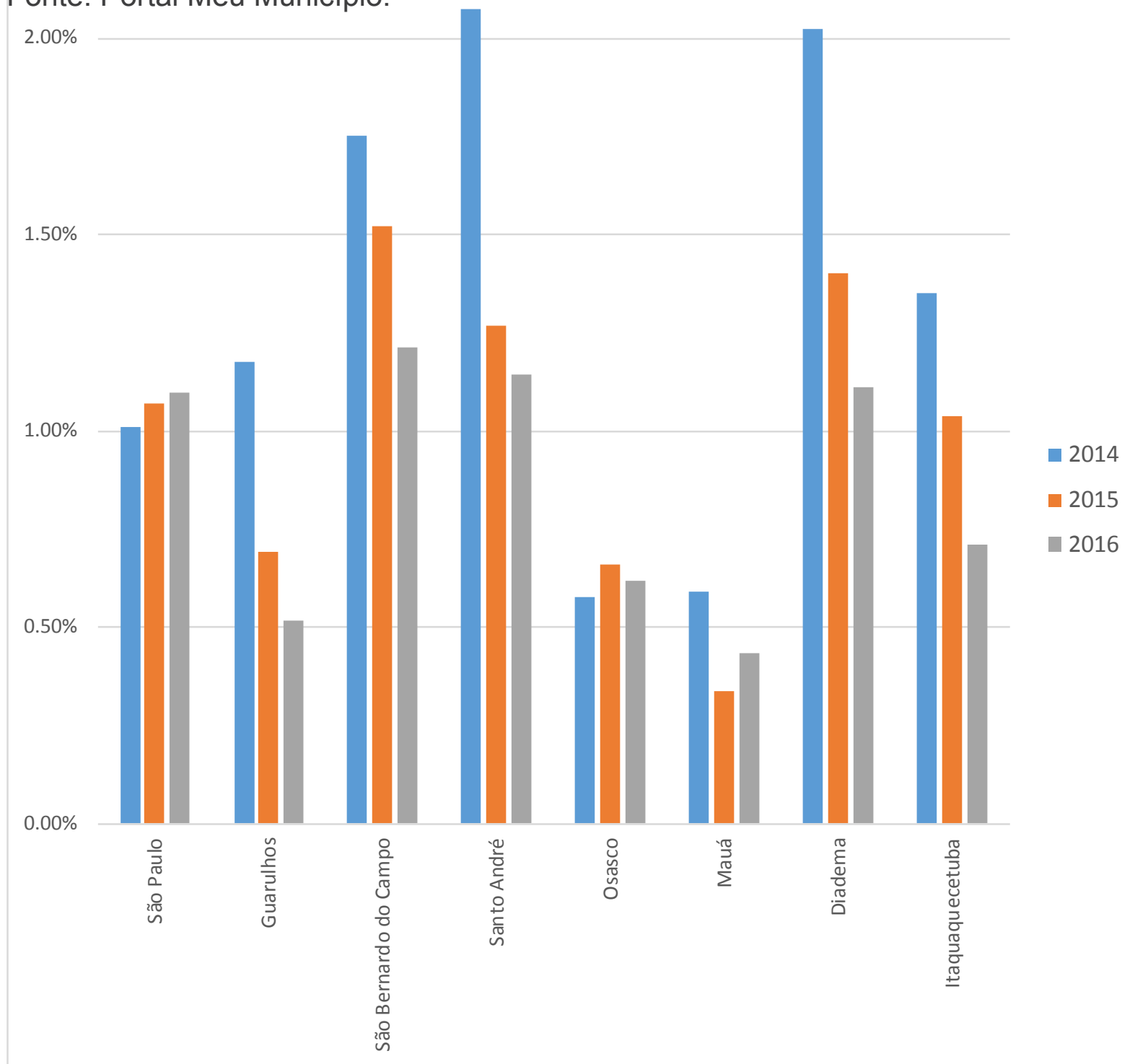


Algumas características dos municípios precisam ser ressaltadas para evidenciar as distintas realidades. Por exemplo, a Secretaria Municipal de Cultura de Mauá está estruturada considerando também a juventude. No município de Guarulhos, a juventude e a cultura podem concorrer sob o mesmo orçamento. Considerando que, além das diferenças de estrutura administrativa e organizacional, há também uma grande diferença populacional entre os municípios, a Tabela 4 mostra o valor per capita apenas para o último ano do período analisado (2016). A análise mostra que, apesar de o orçamento municipal da cultura, em termos absolutos, ser maior em São Paulo, quando levamos em consideração a população residente no município, São Bernardo do Campo leva vantagem, tendo $20,4 \%$ a mais de recursos per capita do que a capital paulista. Essa desigualdade na alocação de recursos fica ainda mais evidente quando pegamos os extremos: São Bernardo do Campo possui mais de seis vezes o orçamento per capita da cultura de Carapicuíba.

Tabela 4. Orçamento municipal da cultura. valores absolutos e per capita (2016).

\begin{tabular}{lcc}
\hline \multicolumn{1}{c}{ Município } & $\begin{array}{c}\text { Orçamento municipal da } \\
\text { cultura (2016) em R\$ }\end{array}$ & $\begin{array}{c}\text { Orçamento municipal da } \\
\text { cultura per capita (2016) } \\
\text { em R\$ }\end{array}$ \\
\hline São Paulo & 526.077 .126 & 43,45 \\
São Bernardo do Campo & 43.284 .150 & 52,31 \\
Santo André & 26.066 .250 & 36,44 \\
Guarulhos & 17.853 .150 & 13,23 \\
Diadema & 13.296 .150 & 31,82 \\
Osasco & 12.293 .291 & 17,62 \\
Itaquaquecetuba & 4.334 .400 & 12,02 \\
Mauá & 4.030 .950 & 8,72 \\
Carapicuíba & 3.413 .550 & 8,61 \\
\hline
\end{tabular}

Fonte: Finbra.

Os valores absolutos maiores da Secretaria Municipal de Cultura de São Paulo justificam a existência de editais de fomento para as seguintes frentes: cinema, circo, dança, teatro, música, livros, pintura de rua e radiodifusão comunitária. Além desses há o chamado "Prêmio Zé Renato", que apoia o desenvolvimento teatral, e o Edital "Redes e Ruas", que possui o objetivo de apoiar "propostas de inclusão, cidadania e cultura digital, que deverão prever, dentre o conjunto de atividades, ações a serem desenvolvidas em telecentros, praças do programa WiFi Livre SP e em parceria com os Pontos de Cultura de São Paulo" (Prefeitura de São Paulo, 2017).

Analisando o comportamento específico do município de São Paulo, o Gráfico 2 mostra dois períodos distintos. Entre 2008 e 2013, com exceção do ano de 2011, houve quedas sucessivas na dotação orçamentária da cul- 
tura em termos reais. Nesse primeiro período, a redução entre 2008 e 2013 foi de $28,8 \%$. Já entre 2013 e 2016, houve um au- mento real de $39,4 \%$ na previsão orçamentária da cultura.

Gráfico 2. Orçamento anual da cultura no município de São Paulo (em R $\$$ de 2016)

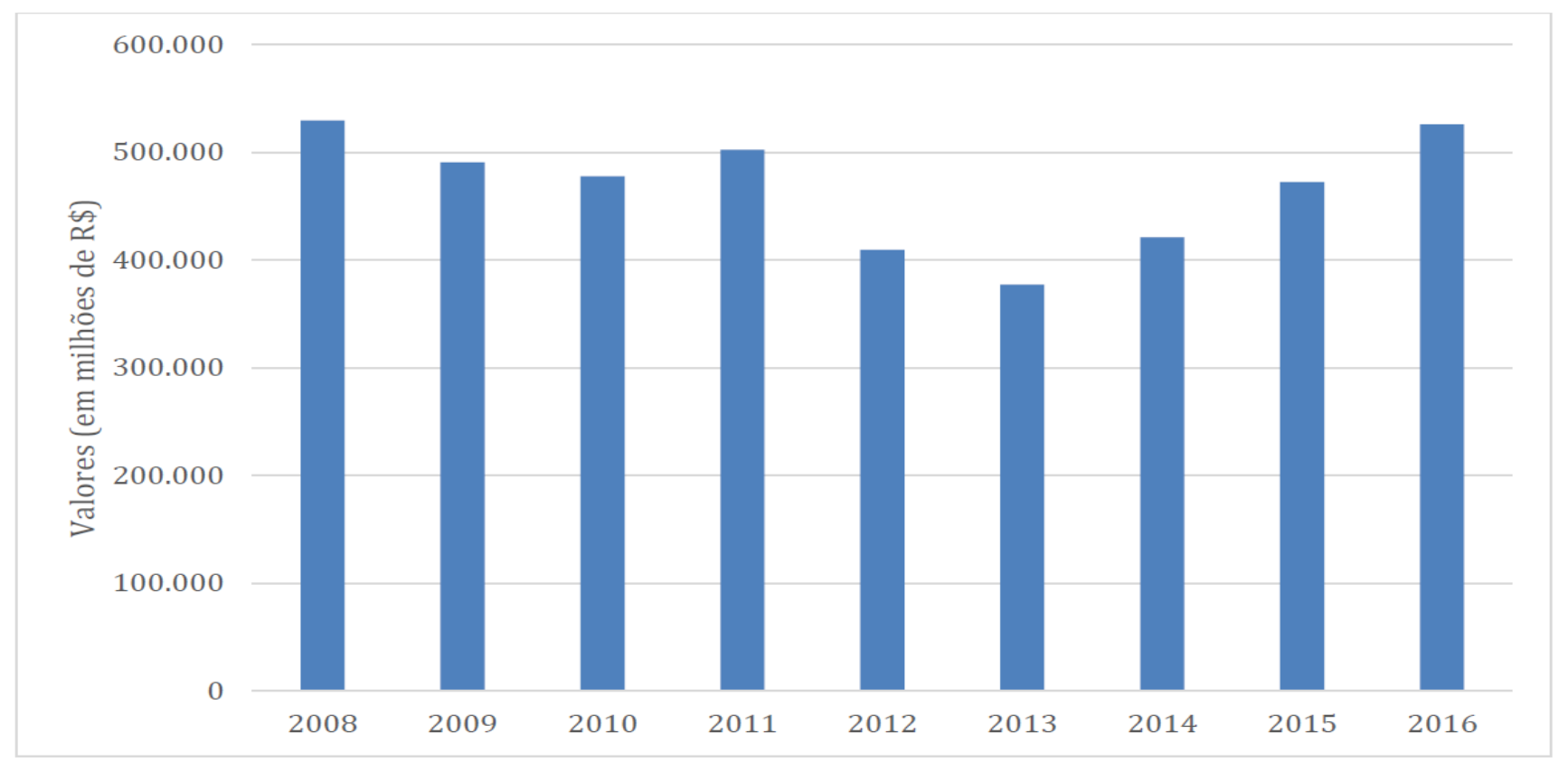

Um dos programas implementados pela Secretaria Municipal de Cultura de São Paulo é o VAl, que possui como principais objetivos o estímulo à criação, ao acesso e à participação do pequeno produtor cultural no desenvolvimento cultural da cidade; a promoção da inclusão de diversos artistas locais na cultura paulistana; e o estímulo às dinâmicas culturais locais. Em 2013, o VAI passou a ser dividido em duas modalidades: VAI I e VAI II. Em 2016, o VAI contemplou 230 projetos em toda a cidade de São Paulo, buscando abarcar a diversidade cultural em atividades como dança, música, teatro, artes cênicas, artes visuais, hip hop, cultura digital, entre outras. No edital de 2017, o primeiro fornece aos projetos até $\mathrm{R} \$ 40 \mathrm{mil}$ e atende grupos de jovens de 18 a 29 anos; e o segundo destina recursos de até $R \$ 80$ mil, atendendo grupos que passaram pelo
VAl 1 e que tenham experiência em ações culturais há pelo menos dois anos.

\section{ENTRE O PREVISTO E O LIQUIDADO: UM DRAMA TAMBÉM DA CULTURA}

Depois que a previsão orçamentária é aprovada pelo Poder Legislativo, começa uma nova etapa: a execução orçamentária. Essa etapa tende a ser bastante complexa para quase todas as estruturas e órgãos da administração pública direta no Brasil. No caso da cultura, isso não é diferente, sendo a inserção de valores condizentes com a realização de atividades culturais com qualidade nos orçamentos municipais o primeiro desafio. O segundo desafio é garantir que, uma vez que os recursos foram adequadamente previstos, eles possam chegar às organizações para que possam executar aquilo que 
foi planejado.

A análise do valor inicialmente planejado e o realmente gasto, entendido como liquidado, demonstra como há diferentes com- portamentos entre os municípios paulistas analisados. O Gráfico 3 mostra o percentual de despesas liquidadas sobre as despesas previstas no final de cada ano.

Gráfico 3. Percentual de despesas liquidadas sobre as despesas previstas por ano

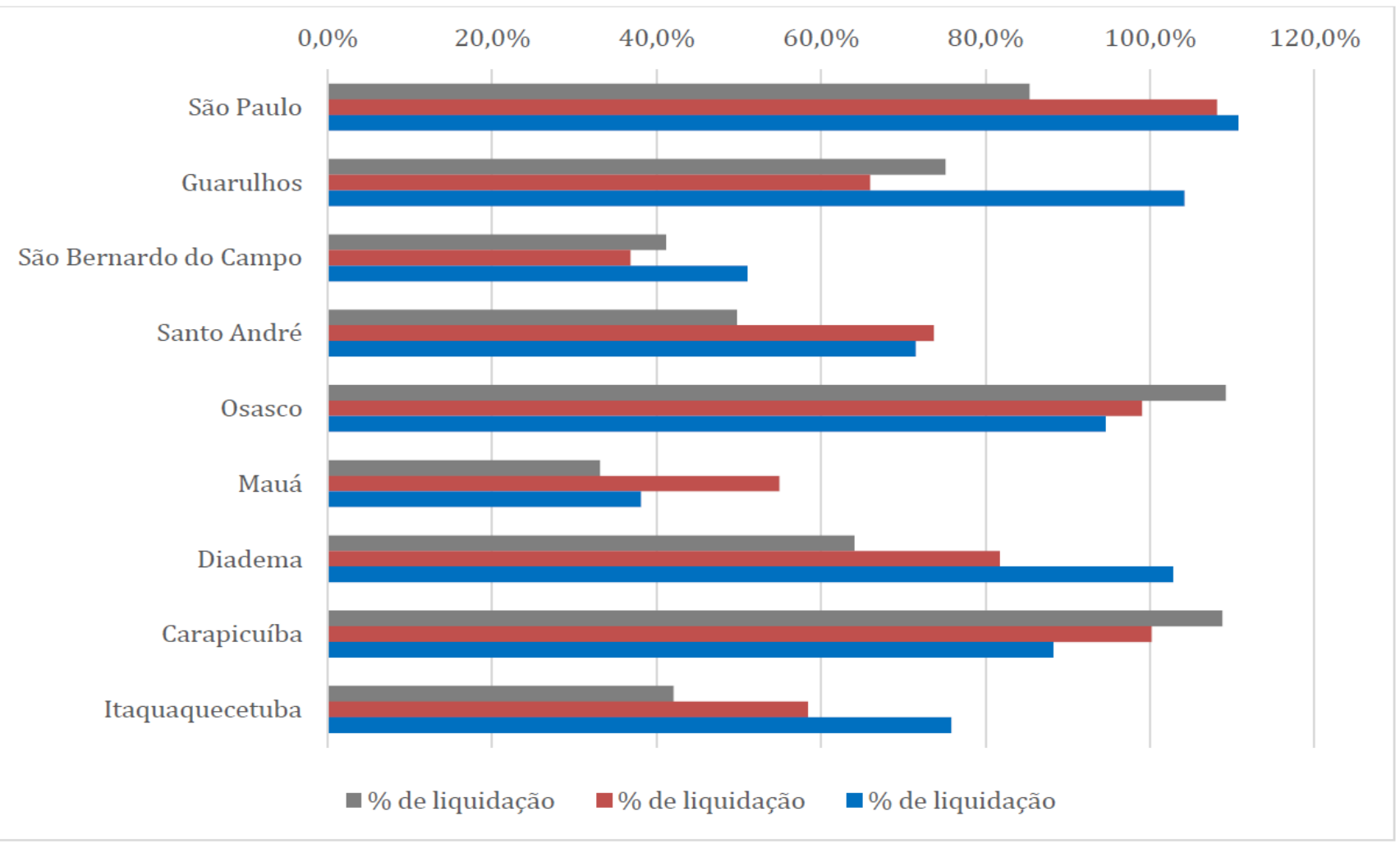

Considerando os municípios da RMSP aqui analisados, podemos dividi-los em quatro grupos. No primeiro, temos São Paulo, Diadema e Itaquaquecetuba, em que o percentual de liquidação melhorou anualmente entre 2014 e 2016. No segundo, temos Guarulhos e São Bernardo do Campo, que tiveram um desempenho pior em 2015, mas se recuperaram em 2016, ultrapassando o percentual de liquidação de 2014. No terceiro, temos Santo André e Mauá, cujo melhor resultado ocorreu em 2015, com uma queda em 2016. E, por fim, temos Osasco e Carapicuíba, cujos resultados vêm piorando desde 2014.
Analisando os dados de 2016, alguns municípios merecem destaque. Em São Paulo, Guarulhos e Diadema, o valor liquidado foi maior do que o previsto inicialmente na LOA. Todos os demais liquidaram menos do que o previsto. Para uma área cujo volume de recursos financeiros disponíveis é sempre um problema, quando um município não consegue executar esse orçamento pequeno, as organizações tendem a sofrer ainda mais. Nesse sentido, Mauá e São Bernardo do Campo parecem ser os mais problemáticos, já que, nos três anos analisados, estiveram perto ou abaixo de $50 \%$ de liquidação dos 
recursos previstos. Entre os anos de 2010 a 2013, a previsão de receitas do município de São Paulo foi inferior, de modo considerável, ao que de fato foi liquidado. Considerando que essa etapa de planejamento é importante para que políticas públicas sejam pensadas e implementadas com maior chance de sucesso, considera-se nessa análise que o município de São Paulo executou seu planejamento de modo aquém do esperado ou externalidades ocorreram junto à pasta de cultura, que fizeram desse setor-alvo de uma série de suplementações orçamentárias.

O cruzamento das informações dos Gráficos 2 e 3 também traz informações importantes. Por exemplo, em São Paulo, as despesas com cultura vêm aumentando, assim como o seu percentual de liquidação. Já em Santo André, além de o percentual de despesas ter sido bastante reduzido, a liquidação foi menor do que 80\% em 2016. $\mathrm{E}$ há casos mais graves, como Mauá, cujo percentual foi o menor entre os municípios analisados, e o mesmo ocorreu com o percentual e liquidação no ano de 2016.

Mas a análise do valor liquidado no final do ano não é suficiente. A prática de limitar 0 empenho (ato de criar a obrigação de realizar um pagamento) das despesas, bem como das movimentações financeiras de uma Prefeitura, é chamado de "contingenciamento." Em um cenário de crise fiscal, essa prática de contingenciar os gastos é recorrente, dado que os governos têm dúvidas sobre se as receitas previstas serão efetivamente executadas. Assim, para garantir o equilíbrio fiscal no final do exercício, e eventualmente alterar as previsões de iniciais, as despesas de custeio e investimen- to tendem a ser adiadas. Ou seja, mesmo quando as despesas liquidadas são maiores do que as previstas no final do ano, se houver contingenciamento ao longo dos meses, o planejamento das organizações é fortemente afetado.

\section{A DINÂMICA ORÇAMENTÁRIA E SEUS EFEITOS NAS ORGANIZAÇÕES CULTU- RAIS}

A pesquisa em campo aponta que a sustentabilidade financeira sempre foi um grande desafio para grupos e iniciativas culturais. Nos últimos anos, editais de fomento à cultura garantiam, por meio do repasse de recursos públicos, que essas organizações culturais conseguissem desempenhar suas atividades. Com menos oferta de recursos, o processo, para ser contemplado por algum edital, torna-se cada vez mais concorrido. Quando vencida essa etapa, outro problema aparece para as organizações culturais entrevistadas: o atraso no repasse de recursos.

Um dos desdobramentos é que o contingenciamento de recursos transforma-se em contingenciamento das atividades programadas para aquele período. Assim, mesmo quando financiadas por meio de políticas públicas, as organizações enfrentam dificuldades que provocam adiamento das atividades, interrupções de algumas delas, ou transformações nas organizações, que passam a buscar outras alternativas para manter o seu funcionamento regular. Se uma das funções do orçamento é possibilitar o planejamento das ações por parte dos agentes implementadores, quando há contingenciamento, o orçamento passa a perder totalmente essa função. 
O caso do VAI, no município de São Paulo, é emblemático. Embora não seja a única forma de sustentabilidade financeira dessas organizações, não há dúvidas de que, quando o projeto ou a iniciativa é contemplada, passa a ser um recurso fundamental para a manutenção - e eventual expansão - das atividades. Mas os dados do Portal da Transparência da Prefeitura Municipal de São Paulo evidenciam a diferença entre o que está planejado e o que, de fato, foi executado: do orçamento previsto para a execução do VAl em 2017 (R\$ 12.865.200,00), apenas $38 \%$ dele $(R \$ 4.889 .917,00)$ constava como pago, em 31 de outubro. Ou seja, faltando dois meses para o fim o ano, ainda havia uma grande parcela de recursos para ser empenhada, liquidada e paga. E atrasos como esses geram impacto direto nas organizações e na execução das atividades planejadas.

As nossas entrevistas com organizações culturais da região mostram que, a partir do cenário de restrição orçamentária, é perceptível uma busca por múltiplas estratégias de sobrevivência. A frase "A falta de dinheiro não pode bloquear a cultura" é um dos argumentos utilizados pelos coletivos de cultura para explicar como ocorrem as estratégias de sobrevivência. A motivação de seguir com os trabalhos realizados, mesmo diante da dificuldade de financiamento e o escopo inicialmente pensado reduzido, tem gerado a busca diferentes formas de sobrevivência financeira, que incluem financiamentos privados e adoção de novas estratégias, como a venda de serviços ou produtos culturais. Foi possível observar que algumas organizações mudaram inclusive o rumo organizacional a fim de se profissionalizar e melhor se adaptar ao campo. Com isso, artistas de formação passaram a ser captadores de recursos, "gerentes de marketing" e "relações públicas" das organizações, além de agentes culturais.

Outra estratégia de lidar com as finanças públicas é a participação dos grupos culturais nas audiências públicas, espaço de debate sobre o orçamento e a priorização de recursos, junto com vereadores ou deputados e representantes da sociedade civil organizada. Há, ainda, a estratégia de fortalecimento da rede de organizações para que a realização das atividades - com ou sem recurso previamente alocado - possa ser mantida. Alguns entrevistados mencionam essa estratégia como "progresso compartilhado", pois é por meio da ação em rede que as organizações se conectam, trocam informações e mantêm as ações em curso.

\section{CONSIDERAÇÕES FINAIS}

O debate sobre o financiamento público de políticas sociais segue presente no cotidiano da administração pública brasileira. Em um cenário de crise econômica e fiscal, ele fica ainda mais premente. Algumas áreas, como saúde, educação e assistência social, por possuírem patamares mínimos de gastos constitucionalmente definidos e/ou sistemas nacionais de políticas públicas, estão um pouco mais "protegidas". Já outras áreas, como cultura e esporte, tornam-se ainda mais vulneráveis nesse momento, dado que, em um eventual conflito distributivo, acabam sendo frequentemente preteridas. A situação financeira dos municípios brasileiros agrava ainda mais o quadro.

Analisando os 10 maiores municípios da RMSP - totalizando mais de 17 milhões de 
pessoas - este trabalho buscou resgatar 0 debate sobre o papel que o financiamento público municipal tem para o planejamento da política pública de cultura na região, a partir da análise das LOAs municipais, das bases de dados sobre execução orçamentária e de entrevistas com gestores de organizações culturais do município de São Paulo.

Um dos limites deste artigo foi devido à dificuldade de encontrar dados sistematizados e específicos sobre a área de cultura, tanto nos portais que consolidam as informações públicas como no Finbra e no próprio Ministério da Cultura. Outro limite foi a ausência de dados disponíveis para a construção e análise da série temporal de 2008 até 2016, como inicialmente planejado. Nesse sentido, chama a atenção a falta de transparência de alguns dos municípios selecionados, descumprindo a legislação vigente no País e dificultando os mecanismos de accountability por parte da população em geral.

De toda forma, os casos analisados mostram que a cultura pode ser uma "função" em termos orçamentários. No caso dos municípios brasileiros, os dados da Munic (IBGE, 2006) mostravam que apenas 4,2\% possuíam secretaria municipal exclusiva para a área de cultura, chegando em 20,4\% segundo a Munic (IBGE, 2014). Enquanto $12,6 \%$ a cultura era um setor subordinado a outra secretaria e $72 \%$ era uma secretaria municipal em conjunto com outras políticas em 2006, esses valores passam a $15,9 \%$ e $57,3 \%$, respectivamente. Assim, mesmo com crescimento da relevância da pasta como tema único, em municípios menores, a cultura ainda é entendida como uma "subfunção".
Diante das baixas execuções orçamentárias e dos contingenciamentos de recursos ao longo do ano, é possível especular que o orçamento municipal passa a ser "sem-função", uma vez que a indefinição sobre a chegada - e o momento dessa chegada dificulta a implementação de qualquer planejamento das organizações culturais. Dada a importância dos mecanismos de repasse de recursos municipais para cultura, refletida no cotidiano nas organizações culturais, a instabilidade de recursos aumenta a disputa no campo, tornando a busca pela sustentabilidade financeira dos grupos culturas um assunto cada vez mais recorrente.

Nesse cenário, reforça-se a necessidade de discutir a mobilização social em torno do planejamento orçamentário, conforme aponta a parte qualitativa da pesquisa, mas também repensar as formas de financiamento e atuação. Ainda em termos de políticas culturais, é relevante apontar que a capacidade de articulação dos agentes que atuam com a cultura e seu grau de influência transversal, entendidos aqui como o resultado do encontro dos pares, podem gerar algo novo e singular no território, deve ser levado em consideração.

Partindo do pressuposto de que a cultura é capaz de interferir na economia, especialmente por meio de arranjos territoriais e de externalidades positivas, torna-se relevante incorporar a capacidade que as diferentes formas, individual ou coletivamente, possuem para colaborar com o desenvolvimento local. Assim, mesmo que o poder público tenha dificuldades no financiamento da cultura, a escolha estratégica por esse segmento pode criar um caminho para o fortalecimento da economia local, por meio da 
geração de renda e de postos de trabalhos, que podem ajudar a diminuir a vulnerabilidade financeira do próprio município.

\section{REFERÊNCIAS}

Barbosa da Silva, F. A. (2004). Financiamento cultural: Situação atual e questões para reflexão. Recuperado de http://www.ipea. gov.br

Bille, T., \& Schulze, G. (2006). Culture in urban and regional development. In V. Ginsburg, \& D. Throsby, Handbook of the economics of arts and culture (pp. 1051-1099). Amsterdan, The Netherlands: Elsevier.

Einarsson, A. (2008). The economic impact of public cultural expenditures on creative industries under increasing globalization. Bifröst Journal of Social Science, 227-46.

Finanças do Brasil. (2018). Relatório Finanças do Brasil, produzido pela Secretaria do Tesouro Nacional - STN. Recuperado de http://www.stn.fazenda.gov.br/estatistica/ est_estados

Frey, B. (1994). Art: The economic point of view. In A. Peacock, \& I. Rizzo, Cultural economics and cultural policies (pp. 3-16). Berlin, Germany: Springer.

Frey, B. (1995). Public support for the arts in a direct democracy. In B. Frey, Arts and economics: Analysis \& cultural policy (pp. 115-129). Berlin, Germany: Springer-Verlag. Fundação Getulio Vargas. (2017). Estação de Pesquisa M'Boi Mirim. Recuperado de http://ceapg.fgv.br/m-boi/sobre-estacao

Giacomoni, J. (2010). Orçamento público.
São Paulo, SP: Atlas.

Giambiagi, F. \& Além, A. C. (2010). Finanças públicas: Teoria e prática no Brasil. São Paulo, SP: Campus.

Gibson, L., \& Stevenson, D. (2004). Urban space and the uses of culture. International Journal of Cultural Policy, 10(1), 1-4. doi:10.1080/1028663042000212292

Håkonsen, L., \& Løyland, K. (2016, November). Local government allocation of cultural services. Journal of Cultural Economics, 40(4), 487-528. doi:10.1007/s10824-0159255-4

Instituto Brasileiro de Geografia, Estatística. (2006). Coordenação de População, \& Indicadores Sociais. Perfil dos municípios brasileiros 2006: Pesquisa de informações básicas municipais. /BGE. Recuperado de https:// ww2.ibge.gov.br/home/estatistica/economia/ perfilmunic/2006/default.shtm

Instituto Brasileiro de Geografia, Estatística. (2014). Coordenação de População, \& Indicadores Sociais. Perfil dos municípios brasileiros 2014: Pesquisa de informações básicas municipais. IBGE. Recuperado de https://ww2.ibge.gov.br/home/estatistica/ economia/perfilmunic/2014/default.shtm

Kuti, E., \& Marschall, M. (1992). Cultural goods and their positive externalities. World Futures: The Journal of New Paradigm Research, 33(1-3), 181-187. doi:10.1080/02604027.1 992.9972280

Lei n. 4.320, de 17 de março de 1964. Institui normas gerais de direito financeiro para elaboração e controle dos orçamentos e balan- 
ços da União, dos Estados, dos Municípios e do Distrito Federal. Presidência da República. Recuperado de http://www.planalto. gov.br/Ccivil_03/leis/L4320.htm

Machado, A. F., Rabelo, A., \& Moreira, A. G. (2004). Specificities of the artistic cultural labor market in Brazilian metropolitan regions between 2002 and 2010. Journal of Cultural Economics, 38(3), 237-251. doi:10.1007/ s10824-013-9210-1

Mello, T., Gorgulho, L. F., Goldenstein, M., Alexandre, P. V. M. (2009). A economia da cultura, o BNDES e o desenvolvimento sustentável. BNDES Setorial, (30), 299-355.

Michetti, M. (2016). A definição privada do bem público: $A$ atuação de institutos empresariais na esfera da cultura. Caderno $\mathrm{CRH}, 29(78)$, 513-534. doi:10.1590/s010349792016000300007

Ministério da Cultura. (2016). Sobre projetos e orientações aos proponentes. Recuperado de http://www.cultura.gov.br/apoio-a-projetos

Musgrave, R. (1959). The theory of public fi- nance. New York, USA: McGraw Hill.

Organização das Nações Unidas. (1948). Declaração Universal dos Direitos Humanos. Recuperado de http://www.onu-brasil. org.br/documentos_direitoshumanos. php

Premchand, A. (1983). Government budgeting and expenditure controls. Washington DC, USA: International Monetary Fund.

Rizzo, I., \& Throsby, D. (2006). Cultural heritage: Economic analysis and public policy. In V. Ginsburg, \& D. Throsby, (Eds.), Handbook of the economics of art and culture (Vol. 1). North-Holland, Netherlands: Elsevier.

Rubim, A. (2013). Políticas culturais do governo Lula. Revista Lusófona de Estudos Culturais, 1(1), 224-242.

Scott, A. J. (1997). The cultural economy of cities. International Journal of Urban and Regional Research, 21, 323-339. doi:10.1111/1468-2427.00075

Throsby, D. (2010). The economics of cultural policy. Cambridge, USA: Cambridge University Press. 